\title{
Fatal outcome in a Hispanic woman with moyamoya syndrome and Graves' disease
}

\author{
Correspondence \\ should be addressed \\ Julian Choi', Perin Suthakar² and Farbod Farmand ${ }^{1}$ \\ Departments of ${ }^{1}$ Internal Medicine and ${ }^{2}$ Neurology, Arrowhead Regional Medical Center, Colton, California, USA \\ to J Choi \\ Email \\ Julian.J.Choi@gmail.com
}

\section{Summary}

We describe the case of a young Hispanic female who presented with thyrotoxicosis with seizures and ischemic stroke. She was diagnosed with a rare vasculopathy - moyamoya syndrome. After starting antithyroid therapy, her neurologic symptoms did not improve. Acute neurosurgical intervention had relieved her symptoms in the immediate post-operative period after re-anastomosis surgery. However, 2 post-operative days later, she was found to be in status epilepticus and in hyperthyroid state. She quickly deteriorated clinically and had expired a few days afterward. This is the second case in literature of a fatality in a patient with moyamoya syndrome and Graves' disease. However, unlike the other case report, our patient had undergone successful revascularization surgery. We believe her underlying non-euthyroid state had potentiated her clinical deterioration. Case studies have shown positive correlation between uncontrolled hyperthyroidism and stroke-like symptoms in moyamoya syndrome. Mostly all patients with these two disease processes become symptomatic in marked hyperthyroid states. Thus, it may be either fluctuations in baseline thyroid function or thyrotoxicosis that potentiate otherwise asymptomatic moyamoya vasculopathy.

\section{Learning points:}

- Awareness of the association between Graves' disease and moyamoya syndrome in younger patients presenting with stroke-like symptoms.

- Obtaining euthyroid states before undergoing revascularization surgery may protect the patient from perioperative mortality and morbidity.

- Although moyamoya disease is usually thought to be genetically associated, there are reports that thyroid antibodies may play a role in its pathogenesis and have an autoimmune link.

- Fluctuations in baseline thyroid function for patients with known Graves' disease may be a potentiating factor in exacerbating moyamoya vasculopathy.

\section{Background}

Moyamoya disease (MMD) is a spontaneous, progressive cerebrovascular disorder characterized by distal stenosis or narrowing of bilateral internal carotid arteries (ICAs), which advances to involve proximal anterior cerebral arteries (ACAs) and middle cerebral arteries (MCAs). Compensatory development of abnormal vasculature known as moyamoya vessels (MMVs) originate near sites of steno-occlusion from lenticulostriate and thalamoperforators, which gives the classic 'puff of smoke' appearance on angiography. Stenotic lesions in MMD are described to have intimal fibrocellular thickening, expansion of elastic lamina and attenuation of media. MMVs vary morphologically as dilated arteries with thin walls or thickened walls with stenosis and are often tortuous. Patients usually present with symptoms of transient ischemic attacks (TIA) or stroke. When radiographic evidence of moyamoya is unilateral or accompanied by a recognized underlying condition, 
patients are classified as having moyamoya syndrome (MMS). Although there are several associated conditions (down syndrome, neurofibromatosis, sickle cell disease, systemic lupus erythematosus, meningitis, irradiation and diabetes mellitus type I), the underlying pathophysiology remains unclear. Graves' disease (GD) is one of the rare associated disorders. GD is an autoimmune disorder in which autoantibodies to thyroid-stimulating hormone (TSH) receptors induce high levels of thyroid hormone in circulation. Thyroid hormones are thought to enhance vascular reactivity and facilitate hemodynamic changes by increasing the sensitivity of the sympathetic nervous system (SNS). Furthermore, thyrotoxicosis is thought to increase cerebral metabolism with increased oxygen consumption and demand, which can lead to impaired cerebral perfusion. In addition, hyperthyroid-associated hypercoagulability may augment ischemic symptoms. This is supported by reports of most patients with MMS and GD presenting with TIA or stroke during a thyrotoxic state or during fluctuations in remission to homeostatic levels of thyroid function. To the best of our knowledge, there are around eighty-seven reported cases in English literature (1). Furthermore, out of those cases, only one documented fatality in a patient with MMS and GD prior to obtaining revascularization surgery. We report a case of MMS with GD in a Hispanic female who presented with right-sided progressive hemiparesis, underwent successful revascularization surgery, complicated by epilepsy and shock and ultimately had a fatal outcome during thyrotoxic state.

\section{Case presentation}

A 31-year-old Hispanic female presented with sudden worsening of progressive right-sided weakness with sluggish and unsteady gait causing several falls in the preceding three weeks. She also had new-onset palpitations with cycles of confusion. Chronic medical conditions included hypertension and Graves' disease (GD) diagnosed one year before by her primary care physician. She had been off propranolol and methimazole five weeks prior to presentation as she ran out of medications. Examination revealed a blood pressure of $179 / 83 \mathrm{mmHg}$, heart rate of $115 \mathrm{bpm}$, sinus tachycardia on ECG, respiratory rate of 20 , afebrile. She was alert and oriented, pupils were $3 \mathrm{~mm}$ and reactive to light, exophthalmos, right facial droop with drooling, a visible goiter was soft and uniform on palpation, non-tender and no nodules or bruits were appreciated. Neurologic exam was remarkable for right arm flaccid paralysis with right leg weakness, absent right shoulder shrug and right-sided hyper-reflexia. However, her left side was unaffected and sensations were intact to light touch and pinprick throughout.

\section{Investigation}

Blood tests and imaging were ordered for suspected thyrotoxicosis and stroke. Thyroid function tests coincided with marked thyrotoxic state (thyroidstimulating hormone (TSH), $<0.005 \mathrm{IU} / \mathrm{mL}$; thyroxine (T4), $23.07 \mu \mathrm{g} / \mathrm{dL}$; total triiodothyronine (TT3), $>651 \mathrm{ng} / \mathrm{dL}$ ). Thyroid ultrasound revealed a diffusely enlarged thyroid gland with heterogeneous echotexture with increased vascular flow. Initial head CT without contrast showed no acute pathology. On the second day of hospitalization, patient had a simple partial seizure that resolved with ativan. Subsequent EEG (prior to revascularization) revealed abnormal left hemispheric slowing that was non-specific for an underlying structural pathology. CTA showed complete occlusion of left ICA at cavernous and supraclinoid portions and severe stenosis of right ICA near bifurcation into MCA and ACA with stenosis of the M1 and $\mathrm{A} 1$ segments with posterior communicating arteries supplying bilateral anterior territories. Brain MRI revealed restricted diffusion abnormality in the left frontal cortex with associated abnormal T2 and FLAIR signal changes consistent with an acute left frontal cortical infarction. Follow-up MRA and MRV showed occlusion of bilateral internal carotid arteries at cavernous segment with occluded right MCA and diminutive left MCA (Figs 1 and 2). Neurointerventionalist evaluation with cerebral angiography found significant bilateral narrowing (left greater than right) from ICA terminus into the proximal segment of MCA to near bifurcation. Primary perfusion was through perforators near ICA-MCA junction, with collateral flow from posterior circulation. Moyamoya syndrome was diagnosed with Suzuki grading stage II for right ICA and stage V for left ICA. Hypercoagulable workup was unremarkable.

\section{Treatment}

Treatment was started on day 1 of admission with propranolol $40 \mathrm{mg}$ po Q8h, and methimazole $10 \mathrm{mg}$ po Q8 h, and antiplatelet therapy. Three days after admission, her neurologic deficits were not improving; we had discussions with the patient and family about treating thyroid dysfunction first to achieve euthyroid state vs undergoing surgical anastomosis. They elected to undergo acute surgery. Bilateral frontotemporoparietal craniotomy 


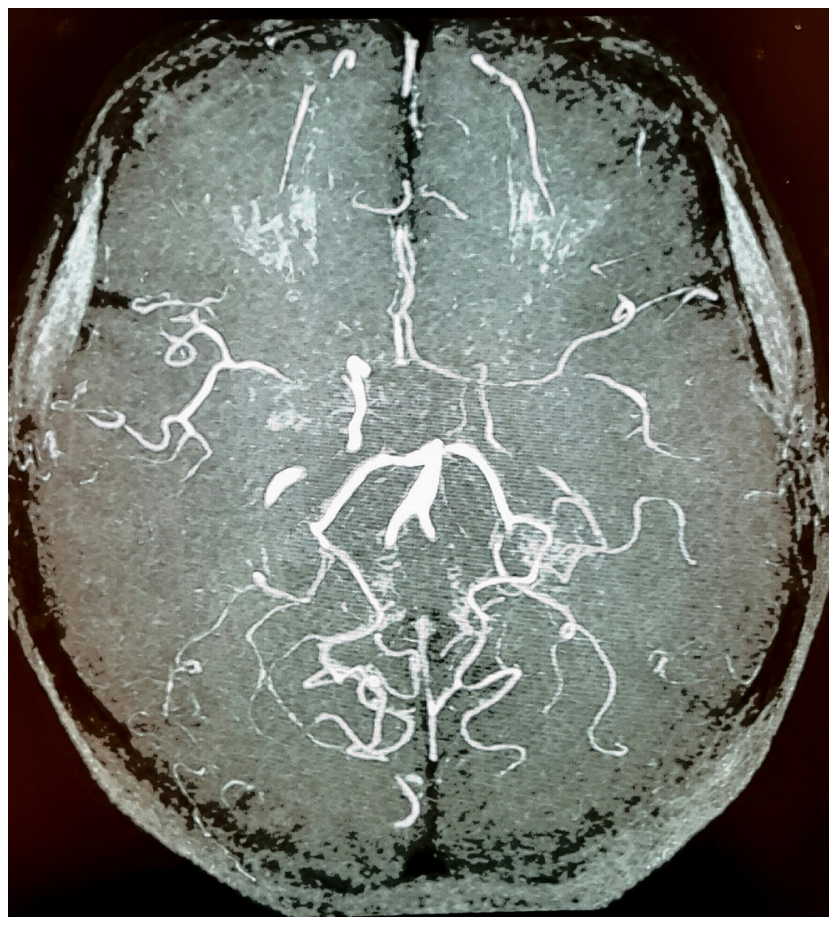

Figure 1

MRA 3D TOF MIP image of the brain showing an occluded right middle cerebral artery, diminutive left middle cerebral artery and bilateral occlusion of internal carotid arteries.

was performed for bilateral encephalomyosynangiosis with no intraoperative complications.

\section{Outcome and follow-up}

The patient was able to move all extremities and follow commands in the immediate postoperative period. Transcranial Doppler was performed indicating mildly increased blood flow velocity without vasospasm in the right middle cerebral, anterior cerebral and left posterior cerebral artery. She was given phenytoin prior to revascularization and continued perioperatively. However,

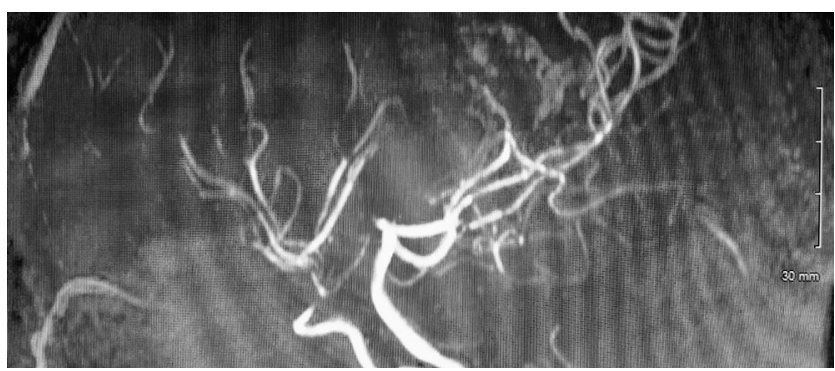

Figure 2

MRA 3D TOF MIP image of the brain showing occlusion of bilateral internal carotid arteries at the cavernous segment. on postoperative day two, the patient had evidence of generalized tonic-clonic seizures while on phenytoin. Lorazepam was given in the acute phase along with loading doses of levetiracetam and valproate. Subsequent 24-h EEG monitoring showed evidence of diffuse reactive slow background activity suggestive of diffuse moderate cortical dysfunction, but no evidence of seizures was noted. However, antiepileptic therapy was maintained at the recommendation of Neurology for inferred seizure activity. On postoperative day five, the patient started to have clinical convulsive activity in the form of tonicclonic seizures and was started on phenobarbital to control her seizures and remitted. Neurology had recommended starting high-dose propofol as bridging therapy toward further antiepileptic therapies. Furthermore, she was found to be septic from a urinary tract infection for which she was treated with non-fluoroquinolone broadspectrum antibiotics. Steroids were precluded due to her underlying infection. During this time, laboratories re-elicited a marked thyrotoxic state. On postoperative day six, the patient became hemodynamically unstable with mixed septic-cardiogenic shock with new-onset atrial fibrillation. She quickly deteriorated, underwent cardiopulmonary arrest and expired.

\section{Discussion}

It is rare for a patient to have MMS and GD and as of July 2016 , to the best of our knowledge, there are about 87 cases in literature $(1,2)$. There was controversy if MMS and GD occurred by coincidence but recent evidence suggests otherwise. Nonetheless, the pathophysiology still remains uncertain.

MMD is hypothesized to be genetically linked, as there is high incidence of familial occurrence with female predominance. Some argue that it is autoimmune mediated, but the absence of immune complexes on blood vessel walls argues against it and favors the presence of a genetic link. However, autoimmunity may contribute a common pathogenesis. There are reports of elevated levels of thyroid antibodies detected in some patients presenting with signs of ischemia secondary to moyamoya disease, even without impaired thyroid functionality (3). Given the increased presence of T-cells within affected vessels, the possibility for T-cell-mediated cross-reactivity between TSH receptor antibodies, and antigens within the cerebral arteries, as well as impaired T-cell function may be a common pathogenetic factor in both diseases $(4,5$, $6,7)$. Colleran et al. (8) has elicited a positive correlation between elevated thyroxine levels with increased 
homocysteine and methylmalonic acid levels. This infers an association among atherosclerosis, GD and MMD. This relationship suggests that pathological changes of GD may influence the susceptibility to moyamoya vasculopathy. Specifically, hyperthyroid states may be an initiating factor of otherwise asymptomatic MMS.

Several reports hypothesize that elevated levels of circulating free thyroxine stiffen arterial walls and decrease the reactivity of the vessels to changes in blood flow. Cerebral oxygen consumption also increases, whereas cerebral perfusion decreases due to elevated sympathetic tone and subsequent vasoconstriction $(5,6$, $1,7)$. Smooth muscle and endothelium of vasculature are influenced by excess circulating thyroid hormones such that the combination of stiffened arteries seen in GD and the significant stenosis observed in MMD predisposes affected individuals to ischemic events and may even pose greater risk than either disease alone $(5,1,9,10)$. In other words, during marked hyperthyroid states, cerebral oxygen demand increases, whereas perfusion decreases and exceeds the compensatory mechanism offered by moyamoya collateral vessels. It would make sense that patients with MMS and GD mostly present with symptoms of stroke during hyperthyroid states. Li et al. (4) report that 18 of 21 of their patients with known MMD and GD showed MMD symptoms in only hyperthyroid states.

As with our case, the patient presented to us in a thyrotoxic state after she had stopped taking antithyroid therapy for 5 weeks. Even after successful revascularization, she suffered from relapsing seizure activity, all during a marked hyperthyroid state. Cause of mortality remains difficult to ascertain as it is complicated by mixed septiccardiogenic shock. Retrospectively, it may have been prudent to correct her thyroid function to euthyroid before undergoing revascularization surgery to decrease the risk of perioperative complications (6). A more aggressive medical approach may have benefitted her clinical course. Along with methimazole and propranolol, an iodine solution could have been given to take advantage of the Wolff-Chaikoff effect to decrease iodine organification. Steroid therapy could have been adjunctive as well, but precluded in her case due to underlying urosepsis.

Recent report by Shah et al. (2) describes 8 patients with GD and MMS (7/8 being Latin American). All of their patients presented with ischemic strokes similar to our patient. Their literature review of 79 cases found no significant difference between conservative medical therapy alone and medical therapy with neurosurgical intervention. This suggests that an aggressive medical approach to obtain euthyroid state should be the first-line approach for patients with MMS and uncontrolled GD prior to considering neurosurgical prophylaxis.

Im et al. (10) also describe a patient who had perioperative stroke-like syndrome during hyperthyroid state after revascularization surgery. Nonetheless, once euthyroid, surgical intervention currently remains the gold standard as 50\% of those treated with antithyroid therapy relapse into hyperthyroid state (5). The only other reported fatality comes from Hsu et al. (11). They describe a patient who underwent radioablation therapy and was found to be in a marked hyperthyroid state that also had rapid deterioration and found to have minimal collateral vessels. However, their patient did not have a chance to undergo revascularization due to her quick clinical decline.

Lastly, rather than hyperthyroid state alone, it is also plausible that fluctuations from baseline thyroid function induce ischemic symptoms in otherwise asymptomatic MMD (4, 1, 7). More than hyperthyroid state alone, fluctuations in thyroid function could have been the culprit that led to her fatality. As she was septic from a urinary tract infection, her baseline hyperthyroid state likely was exacerbated by an inflammatory sympathetic response from her infection and possibly potentiated her unrelenting seizure activity.

It may be prudent that patients with MMS and GD obtain euthyroid states prior to considering revascularization surgery especially in those who present in thyrotoxicosis or fluctuations from baseline. In addition, providers should be aware of the association between GD and MMS in those who present with stroke symptoms. GD should be managed carefully during the perioperative period to give patients the highest possibility in obtaining successful outcomes.

Declaration of interest

Dr Julian Choi has no conflict of interest or financial disclosure relevant to the topic of the submitted manuscript. Dr Farbod Farmand has no conflict of interest or financial disclosure relevant to the topic of the submitted manuscript. Dr Perin Suthakar has no conflict of interest or financial disclosure relevant to the topic of the submitted manuscript.

\section{Funding}

This research did not receive any specific grants from any funding agency in the public, commercial or not-for-profit sector.

\section{Patient consent}

The patient is deceased, and there have been attempts made via telephone to contact relatives. However, the phone number we have on file has been changed and thus our attempts have been unsuccessful. 


\section{Author contribution statement}

Julian Choi, provided substantial contributions to conception and design, acquisition of data or analysis and interpretation of data. Julian Choi, Farbod Farmand, Perin Suthakar, drafted the article or revised it critically for important intellectual content. Julian Choi, Farbod Farmand, Perin Suthakar, gave final approval of the version of the article to be published. Julian Choi, Farbod Farmand, Perin Suthakar, agree to be accountable for all aspects of the work in ensuring that questions related to the accuracy or integrity of any part of the work are appropriately investigated and resolved.

\section{References}

1 Chen J, Lei D, He M, Sun H, Liu Y, Zhang H, You C \& Zhou LX 2015 Clinical features and disease progression in moyamoya disease patients with Graves disease. Journal of Neurosurgery 123 848-855. (doi:10.3171/2014.10.JNS141140)

2 Shah NH, Khandelwal P, Gordon-Peru G, Shah A, Barbarite E, Ortiz G \& Forteza AM 2016 Acute thyrotoxicosis of Graves disease associated with moyamoya vasculopathy and stroke in Latin American women: a case series and review of the literature. World Neurosurgery 92 95-107. (doi:10.1016/j.wneu.2016.04.122)

3 Kim SJ, Heo KG, Shin HY, Bang OY, Kim GM, Chung CS, Kim KH, Jeon P, Kim JS, Hong SC, et al. 2009 Association of thyroid autoantibodies with Moyamoya-type cerebrovascular disease: a prospective study. Stroke 41 173-176. (doi:10.1161/ STROKEAHA.109.562264)
4 Li D, Yang W, Xian P, Liu P, Bao X, Zong R \& Duan L 2009 Coexistence of moyamoya and Graves' diseases: the clinical characteristics and treatment effects of 21 Chinese patients. Clinical Neurology and Neurosurgery 115 1647-1652. (doi:10.1016/j.clineuro.2013.02.018)

5 Endo H, Fujimura M, Niizuma K, Shimizu H \& Tominaga T 2010 Efficacy of revascularization surgery for moyamoya syndrome associated with Graves' disease. Neurologia Medico-Chirurgica 50 977-983. (doi:10.2176/nmc.50.977)

6 Ohba S, Nakagawa T \& Murakami H 2011 Concurrent Graves' disease and intracranial arterial stenosis/occlusion: special considerations regarding the state of thyroid function, etiology, and treatment. Neurosurgical Review 34 297-304. (doi:10.1007/s10143-011-0311-z)

7 Tendler BE, Shoukri K, Malchoff C, MacGillivray D, Duckrow R, Talmadge T \& Ramsby GR 1997 Concurrence of Graves' disease and dysplastic cerebral blood vessels of the moyamoya variety. Thyroid $\mathbf{7}$ 625-629. (doi:10.1089/thy.1997.7.625)

8 Colleran KM, Ratliff DM \& Burge MR 2003 Potential association of thyrotoxicosis with vitamin B and folate deficiencies, resulting in risk for hyperhomocysteinemia and subsequent thromboembolic events. Endocrine Practice 9 290-295. (doi:10.4158/EP.9.4.290)

9 Lee R, Sung K, Park YM, Yu JJ, Koh YC \& Chung S 2009 A case of Moyamoya disease in a girl with thyrotoxicosis. Yonsei Medical Journal 50 594. (doi:10.3349/ymj.2009.50.4.594)

10 Im S, Oh CW, Kwon O, Kim JE \& Han DH 2005 Moyamoya disease associated with Graves disease: special considerations regarding clinical significance and management. Journal of Neurosurgery 102 1013-1017. (doi:10.3171/jns.2005.102.6.1013)

11 Hsu SW, Chaloupka JC \& Fattal D 2006 Rapidly progressive fatal bihemispheric infarction secondary to Moyamoya syndrome in association with Graves thyrotoxicosis. American Journal of Neuroradiology 27 643-647.

Received in final form 4 July 2016 Accepted 24 October 2016 